Proyecciones Journal of Mathematics

Vol. 34, No 3, pp. 277-296, September 2015.

Universidad Católica del Norte

Antofagasta - Chile

\title{
Ramanujan's fifth order and tenth order mock theta functions - a generalization
}

\author{
Bhaskar Srivastava \\ University of Lucknow, India \\ Received : January 2015. Accepted : July 2015
}

\begin{abstract}
A generalization of Ramanujan's fifth order and tenth order mock theta functions is given. It is shown that these belong to the family of $F_{q}$-functions. Using the properties of $F_{q}$-functions, relationship is given between these generalized fifth order mock theta functions of the first group with the generalized functions of the second group. The same is done for the generalized functions of the tenth order. q-Integral representation and multibasic expansions are also given.
\end{abstract}

Subjclass : [2000]33D15.

Keywords : Mock theta function, q-Multibasic expansion, q-Integral. 


\section{Introduction}

Early in 1920, January 12, 1920 to be exact, three months before his death, S. Ramanujan wrote his last letter to G.H. Hardy [7, pp.354-355]. He wrote "I discovered very interesting functions recently which I call 'Mock' $\theta$ functions. Unlike the 'False' $\theta$-functions (studied partially by Prof. Rogers in his paper [8]) they enter into mathematics as beautifully as the ordinary $\theta$-functions. I am sending you with this letter some examples". He then gave a list of seventeen functions, four of order three, ten of order five and three of order seven together with the identities that they satisfy.

G.N. Watson was the first to write papers on the mock theta functions $[9,10]$. The first of these is Watson's Presidential Address to the London Mathematical Society in 1935. The title was "The Final Problem: An Account of the Mock Theta Functions". In these two papers Watson proved most of the identities found in the letter of Ramanujan. The first paper considers only the third order mock theta functions. It also gives three new third order mock theta functions.

Mock theta functions of order five and seven were difficult. The fifth order mock theta functions are in two groups and Ramanujan claimed that no relation exists between mock theta functions of the first group with mock theta functions of the second group. We have given a generalization of the fifth order mock theta functions. Our aim is to give relations between the generalized functions of the first group with the generalized functions of the second group. A generalization of the Ramanujan's tenth order mock theta functions and relations between these generalized functions is also given. In section 3 , we show that these generalized functions belong to the family of $F_{q}$-functions.

By using the difference operator we develop relations between the generalized functions of the two groups, this is done in section 4 .

In section 5 we represent these functions as $q$-Integrals and in section 6 multibasic expansion is given for these generalized functions. In section 7 we show that the generalized tenth order mock theta functions are $F_{q^{-}}$ functions and in section 8 relations among these generalized functions are given. In section 9 we represent these generalized tenth order mock theta function as $q$-Integrals and in section 10 we give multibasic expansion for these generalized functions.

\section{Definition of $F_{q}$-functions}

The functions which satisfy the functional equation 


$$
D_{q, z} F(z, \alpha)=F(z, \alpha+1)
$$

where

$$
z D_{q, z} F(z, \alpha)=F(z, \alpha)-F(z q, \alpha)
$$

are called $F_{q}$-functions.

Throughout the paper we use the following usual basic hypergeometric notations :

$$
\begin{aligned}
& \text { For }\left|q^{k}\right|<1 \text {, } \\
& \left(a ; q^{k}\right)_{n}=(1-a)\left(1-a q^{k}\right) \ldots \ldots .\left(1-a q^{k(n-1)}\right), n \geq 1 \\
& \left(a ; q^{k}\right)_{0}=1 \text {, } \\
& \left(a ; q^{k}\right)_{\infty}=\prod_{j=0}^{\infty}\left(1-a q^{k j}\right) .
\end{aligned}
$$

For convenience we shall write

$$
\left(a_{1}, a_{2}, \ldots \ldots, a_{m} ; q^{k}\right)_{n}=\left(a_{1} ; q^{k}\right)_{n}\left(a_{2} ; q^{k}\right)_{n} \ldots \ldots \ldots \ldots\left(a_{m} ; q^{k}\right)_{n} .
$$

When $k=1$, we usually write $(a)_{n}$ and $(a)_{\infty}$ insteat of $(a ; q)_{n}$ and $(a ; q)_{\infty}$, respectively.

\section{Definition of fifth order and tenth order mock theta func- tions and their generalization}

Ramanujan's ten fifth order mock theta functions are as follows :

$$
\begin{aligned}
f_{0}(q) & :=\sum_{n=0}^{\infty} \frac{q^{n^{2}}}{(-q)_{n}}, & \varphi_{0}(q):=\sum_{n=0}^{\infty} q^{n^{2}}\left(-q ; q^{2}\right)_{n}, \\
\psi_{0}(q):=\sum_{n=0}^{\infty} q^{(n+1)(n+2) / 2}(-q)_{n}, & & F_{0}(q):=\sum_{n=0}^{\infty} \frac{q^{2 n^{2}}}{\left(q ; q^{2}\right)_{n}} \\
\chi_{0}(q) & :=\sum_{n=0}^{\infty} \frac{q^{n}}{\left(q^{n+1}\right)_{n}}, &
\end{aligned}
$$

and

$$
\begin{array}{ll}
f_{1}(q):=\sum_{n=0}^{\infty} \frac{q^{n^{2}+n}}{(-q)_{n}}, & \varphi_{1}(q):=\sum_{n=0}^{\infty} q^{(n+1)^{2}}\left(-q ; q^{2}\right)_{n} \\
\psi_{1}(q):=\sum_{n=0}^{\infty} q^{\frac{n^{2}+n}{2}}(-q)_{n}, & F_{1}(q):=\sum_{n=0}^{\infty} \frac{q^{2 n^{2}+2 n}}{\left(q ; q^{2}\right)_{n+1}} \\
\chi_{1}(q):=\sum_{n=0}^{\infty} \frac{q^{n}}{\left(q^{n+1}\right)_{n+1}} . &
\end{array}
$$


Ramanujan's four tenth order mock theta functions are as follows :
$\Phi(q):=\sum_{n=0}^{\infty} \frac{q^{n(n+1) / 2}}{\left(q ; q^{2}\right)_{n+1}}$,
$\Psi(q):=\sum_{n=0}^{\infty} \frac{q^{(n+1)(n+2) / 2}}{\left(q ; q^{2}\right)_{n+1}}$,
$X(q):=\sum_{n=0}^{\infty} \frac{(-1)^{n} q^{n^{2}}}{(-q ; q)_{2 n}}$,
$\chi(q):=\sum_{n=0}^{\infty} \frac{(-1)^{n} q^{(n+1)^{2}}}{(-q ; q)_{2 n+1}}$.

We define generalization of Ramanujan's ten fifth order mock theta functions as follows :

$$
\begin{aligned}
& f_{0}(t, z, \alpha ; q):=\frac{1}{(t)_{\infty}} \sum_{n=0}^{\infty} \frac{(t)_{n} q^{n^{2}-3 n+n \alpha} z^{2 n}}{(-z ; q)_{n}} \\
& \varphi_{0}(t, z, \alpha ; q):=\frac{1}{(t)_{\infty}} \sum_{n=0}^{\infty} \frac{(t)_{n} q^{n^{2}+n+n \alpha}}{z^{2 n}}\left(-q^{3} / z^{2} ; q^{2}\right)_{n}, \\
& \psi_{0}(t, z, \alpha ; q):=\frac{1}{(t)_{\infty}} \sum_{n=0}^{\infty} \frac{(t)_{n} q^{\frac{n(n+1)}{2}+n \alpha}}{z^{n}}\left(-q^{2} / z ; q\right)_{n-1} \\
& F_{0}(t, z, \alpha ; q):=\frac{1}{(t)_{\infty}} \sum_{n=0}^{\infty} \frac{(t)_{n} q^{2 n^{2}-5 n+n \alpha} z^{4 n}}{\left(z^{2} / q ; q^{2}\right)_{n}} \\
& \chi_{0}(t, z, \alpha ; q):=\frac{1}{(t)_{\infty}} \sum_{n=0}^{\infty} \frac{(t)_{n} q^{n \alpha}(z ; q)_{n}}{\left(z^{2} q^{-1} ; q\right)_{2 n}} \\
& f_{1}(t, z, \alpha ; q):=\frac{1}{(t)_{\infty}} \sum_{n=0}^{\infty} \frac{(t)_{n} q^{n^{2}-2 n+n \alpha} z^{2 n}}{(-z ; q)_{n}} \\
& \varphi_{1}(t, z, \alpha ; q):=\frac{q^{5}}{z^{4}} \frac{1}{(t)_{\infty}} \sum_{n=0}^{\infty} \frac{(t)_{n} q^{n^{2}+3 n+n \alpha}}{z^{2 n}}\left(-q^{3} / z^{2} ; q^{2}\right)_{n}, \\
& \psi_{1}(t, z, \alpha ; q):=\frac{q}{z} \frac{1}{(t)_{\infty}} \sum_{n=0}^{\infty} \frac{(t)_{n} q^{n(n+1) / 2+n \alpha}}{z^{n}}\left(-q^{2} / z ; q\right)_{n}, \\
& F_{1}(t, z, \alpha ; q):=\frac{1}{(t)_{\infty}} \sum_{n=0}^{\infty} \frac{(t)_{n} q^{2 n^{2}-3 n+n \alpha} z^{4 n}}{\left(z^{2} / q ; q^{2}\right)_{n+1}} \\
& \chi_{1}(t, z, \alpha ; q):=\frac{1}{(t)_{\infty}} \sum_{n=0}^{\infty} \frac{(t)_{n} q^{n \alpha}(z ; q)_{n}}{\left(z^{2} q^{-1} ; q\right)_{2 n+1}} .
\end{aligned}
$$

Setting $t=0, \alpha=1$ and $z=q$ these functions reduce to fifth order mock theta functions of Ramanujan. 
Ramanujan's fifth order and tenth order mock theta functions - a ...281

We define a generalization of four tenth order mock theta functions of Ramanujan :

$$
\begin{gathered}
\Phi(t, z, \alpha ; q):=\frac{1}{(t)_{\infty}} \sum_{n=0}^{\infty} \frac{(t)_{n} q^{n(n-3) / 2+n \alpha} z^{n}}{\left(z^{2} / q ; q^{2}\right)_{n+1}}, \\
\Psi(t, z, \alpha ; q):=\frac{z}{(t)_{\infty}} \sum_{n=0}^{\infty} \frac{(t)_{n} q^{n(n-1) / 2+n \alpha} z^{n}}{\left(z^{2} / q ; q^{2}\right)_{n+1}} \\
X(t, z, \alpha ; q):=\frac{1}{(t)_{\infty}} \sum_{n=0}^{\infty} \frac{(-1)^{n}(t)_{n} q^{n^{2}-3 n+n \alpha} z^{2 n}}{\left(-z^{2} / q ; q\right)_{2 n}}, \\
\chi(t, z, \alpha ; q):=\frac{1}{(t)_{\infty}} \sum_{n=0}^{\infty} \frac{(-1)^{n}(t)_{n} q^{n^{2}-n+1+n \alpha} z^{2 n}}{\left(-z^{2} / q ; q\right)_{2 n+1}} .
\end{gathered}
$$

Setting $t=0, \alpha=1$ and $z=q$, these functions redeuce to Ramanujan's tenth order mock theta functions.

\section{Generalized fifth order mock theta functions are $F_{q}$-functions}

We show that our generalized functions satisfy the $F_{q}$-equation (1.1) and so are $F_{q}$-functions.

\section{Theorem 1}

The generalized ten fifth order mock theta functions $f_{0}(t, z, \alpha ; q), \varphi_{0}(t, z, \alpha ; q), \psi_{0}(t, z, \alpha ; q), F_{0}(t, z, \alpha ; q), \chi_{0}(t, z, \alpha ; q), f_{1}(t, z, \alpha ; q)$, $\varphi_{1}(t, z, \alpha ; q), \psi_{1}(t, z, \alpha ; q), F_{1}(t, z, \alpha ; q), \chi_{1}(t, z, \alpha ; q)$ are $F_{q}$-functions.

We give a detailed proof for the function $f_{0}(t, z, \alpha ; q)$ only, the proof for the other functions are similar, so omitted.

\section{Proof}

Applying the difference oprator $D_{q, t}$ to $f_{0}(t, z, \alpha ; q)$, we have

$$
\begin{gathered}
t D_{q, t} f_{0}(t, z, \alpha ; q)=f_{0}(t, z, \alpha ; q)-f_{0}(t q, z, \alpha ; q) \\
=\frac{1}{(t)_{\infty}} \sum_{n=0}^{\infty} \frac{(t)_{n} q^{n^{2}-3 n+n \alpha} z^{2 n}}{(-z ; q)_{n}}-\frac{1}{(t q)_{\infty}} \sum_{n=0}^{\infty} \frac{(t q)_{n} q^{n^{2}-3 n+n \alpha} z^{2 n}}{(-z ; q)_{n}}
\end{gathered}
$$




$$
\begin{gathered}
=\frac{1}{(t)_{\infty}} \sum_{n=0}^{\infty} \frac{(t)_{n} q^{n^{2}-3 n+n \alpha} z^{2 n}}{(-z ; q)_{n}}-\frac{1}{(t)_{\infty}} \sum_{n=0}^{\infty} \frac{(t)_{n} q^{n^{2}-3 n+n \alpha} z^{2 n}\left(1-t q^{n}\right)}{(-z ; q)_{n}} \\
=\frac{t}{(t)_{\infty}} \sum_{n=0}^{\infty} \frac{(t)_{n} q^{n^{2}-3 n+n(\alpha+1)} z^{2 n}}{(-z ; q)_{n}} .
\end{gathered}
$$

So

$$
D_{q, t} f_{0}(t, z, \alpha ; q)=f_{0}(t, z, \alpha+1 ; q) .
$$

Hence $f_{0}(t, z, \alpha ; q)$ is a $F_{q}$-function.

Similarly all other functions in Theorem 1 are $F_{q}$-functions.

\section{Relations between the generalized fifth order mock theta functions of the first group with the generalized fifth order mock theta function of the second group}

In proving the relationship we shall use the property that they are $F_{q^{-}}$ functions, as proved in section 3 .

\section{Theorem 2}

(i) $D_{q, t} f_{0}(t, z, \alpha ; q)=f_{1}(t, z, \alpha ; q)$,

(ii) $D_{q, t}^{2} \varphi_{0}(t, z, \alpha ; q)=\frac{z^{4}}{q^{5}} \varphi_{1}(t, z, \alpha ; q)$,

(iii) $\chi_{0}(t, z, \alpha ; q)=\chi_{1}(t, z, \alpha ; q)-\frac{z^{2}}{q} D_{q, t}^{2} \chi_{1}(t, z, \alpha ; q)$,

(iv) $\psi_{1}(t, z, \alpha ; q)=\frac{q}{z} \psi_{0}(t, z, \alpha ; q)+\frac{q^{2}}{z^{2}} D_{q, t} \psi_{0}(t, z, \alpha ; q)$,

(v) $F_{0}(t, z, \alpha ; q)=\frac{1}{(t)_{\infty}} \sum_{n=0}^{\infty} \frac{(t)_{n} q^{2 n^{2}-5 n+n \alpha} z^{4 n}}{\left(z^{2} / q ; q^{2}\right)_{n+1}}-\frac{z^{2}}{q} F_{1}(t, z, \alpha ; q)$.

\section{Proof of (i)}

$$
\begin{gathered}
D_{q, t} f_{0}(t, z, \alpha ; q)=f_{0}(t, z, \alpha+1 ; q) \\
=\frac{1}{(t)_{\infty}} \sum_{n=0}^{\infty} \frac{(t)_{n} q^{n^{2}-2 n+n \alpha} z^{2 n}}{(-z ; q)_{n}} \\
=f_{1}(t, z, \alpha ; q),
\end{gathered}
$$

which proves Theorem 2 (i) 
Ramanujan's fifth order and tenth order mock theta functions - a ...283

\section{Proof of (ii)}

$$
\begin{gathered}
D_{q, t}^{2} \varphi_{0}(t, z, \alpha ; q)=\varphi_{0}(t, z, \alpha+2 ; q) \\
=\frac{1}{(t)_{\infty}} \sum_{n=0}^{\infty} \frac{(t)_{n} q^{n^{2}+3 n+n \alpha}}{z^{2 n}}\left(-q^{3} / z^{2} ; q^{2}\right)_{n} \\
=\frac{z^{4}}{q^{5}} \varphi_{1}(t, z, \alpha ; q),
\end{gathered}
$$

which proves Theorem 2 (ii).

Proof of (iii)

$$
\begin{aligned}
& \chi_{0}(t, z, \alpha ; q)= \frac{1}{(t)_{\infty}} \sum_{n=0}^{\infty} \frac{(t)_{n} q^{n \alpha}(z ; q)_{n}\left(1-z^{2} q^{2 n-1}\right)}{\left(z^{2} q^{-1} ; q\right)_{2 n+1}} \\
&= \frac{1}{(t)_{\infty}} \sum_{n=0}^{\infty} \frac{(t)_{n} q^{n \alpha}(z ; q)_{n}}{\left(z^{2} q^{-1} ; q\right)_{2 n+1}}-\frac{z^{2}}{q} \frac{1}{(t)_{\infty}} \sum_{n=0}^{\infty} \frac{(t)_{n} q^{n \alpha+2 n}(z ; q)_{n}}{\left(z^{2} q^{-1} ; q\right)_{2 n+1}} \\
&=\chi_{1}(t, z, \alpha ; q)-\frac{z^{2}}{q} D_{q, t}^{2} \chi_{1}(t, z, \alpha ; q)
\end{aligned}
$$

which proves Theorem 2 (iii).

\section{Proof of (iv)}

$$
\begin{gathered}
\psi_{1}(t, z, \alpha ; q)=\frac{q}{z} \frac{1}{(t)_{\infty}} \sum_{n=0}^{\infty} \frac{(t)_{n} q^{n(n+1) / 2+n \alpha}\left(-q^{2} / z ; q\right)_{n}}{z^{n}} \\
=\frac{q}{z} \frac{1}{(t)_{\infty}} \sum_{n=0}^{\infty} \frac{(t)_{n} q^{n(n+1) / 2+n \alpha}\left(-q^{2} / z ; q\right)_{n-1}\left(1+q^{n+1} / z\right)}{z^{n}} \\
=\frac{q}{z} \frac{1}{(t)_{\infty}} \sum_{n=0}^{\infty} \frac{(t)_{n} q^{n(n+1) / 2+n \alpha}\left(-q^{2} / z ; q\right)_{n-1}}{z^{n}}+\frac{q^{2}}{z^{2}} \frac{1}{(t)_{\infty}} \\
\times \sum_{n=0}^{\infty} \frac{(t)_{n} q^{n(n+1) / 2+n \alpha+n}\left(-q^{2} / z ; q\right)_{n-1}}{z^{n}} \\
=\frac{q}{z} \psi_{0}(t, z, \alpha ; q)+\frac{q^{2}}{z^{2}} D_{q, t} \psi_{0}(t, z, \alpha ; q),
\end{gathered}
$$

which proves Theorem 2 (iv). 


$$
\begin{aligned}
& \text { Proof of (v) } \\
& \begin{aligned}
& F_{0}(t, z, \alpha ; q)=\frac{1}{(t)_{\infty}} \sum_{n=0}^{\infty} \frac{(t)_{n} q^{2 n^{2}-5 n+n \alpha} z^{4 n}}{\left(z^{2} / q ; q^{2}\right)_{n}} \\
&=\frac{1}{(t)_{\infty}} \sum_{n=0}^{\infty} \frac{(t)_{n} q^{2 n^{2}-5 n+n \alpha} z^{4 n}\left(1-z^{2} q^{2 n-1}\right)}{\left(z^{2} / q ; q^{2}\right)_{n+1}} \\
&=\frac{1}{(t)_{\infty}} \sum_{n=0}^{\infty} \frac{(t)_{n} q^{2 n^{2}-5 n+n \alpha} z^{4 n}}{\left(z^{2} / q ; q^{2}\right)_{n+1}}-\frac{z^{2}}{q} \frac{1}{(t)_{\infty}} \sum_{n=0}^{\infty} \frac{(t)_{n} q^{2 n^{2}-3 n+n \alpha} z^{4 n}}{\left(z^{2} / q ; q^{2}\right)_{n+1}} \\
&=\frac{1}{(t)_{\infty}} \sum_{n=0}^{\infty} \frac{(t)_{n} q^{2 n^{2}-5 n+n \alpha} z^{4 n}}{\left(z^{2} / q ; q^{2}\right)_{n+1}}-\frac{z^{2}}{q} F_{1}(t, z, \alpha ; q)
\end{aligned}
\end{aligned}
$$

which proves Theorem $2(\mathrm{v})$.

\section{5. $q$-Integral representation for the generalized fifth order mock theta functions}

The $q$-integral was defined by Thomae and Jackon [5, p.19] as $\int_{0}^{1} f(t) d_{q} t=$ $(1-q) \sum_{n=0}^{\infty} f\left(q^{n}\right) q^{n}$.

Limiting case of $q$-beta integral [5, p.19 (1.11.7)] is

$$
\frac{1}{\left(q^{x} ; q\right)_{\infty}}=\frac{(1-q)^{-1}}{(q ; q)_{\infty}} \int_{0}^{1} t^{x-1}(t q ; q) d_{q} t
$$

We use extensively (5.1) to give $q$-integral representation for the generalized functions defined in section 2 .

\section{Theorem 3}

the following equalities hold :
(i) $\quad f_{0}\left(q^{t}, z, \alpha ; q\right)=\frac{(1-q)^{-1}}{(q ; q)_{\infty}} \int_{0}^{1} w^{t-1}(w q ; q)_{\infty} f_{0}(0, z, a w ; q) d_{q} w$
(ii) $\varphi_{0}\left(q^{t}, z, \alpha ; q\right)=\frac{(1-q)^{-1}}{(q ; q)_{\infty}} \int_{0}^{1} w^{t-1}(w q ; q)_{\infty} \varphi_{0}(0, z, a w ; q) d_{q} w$
(iii) $\quad \psi_{0}\left(q^{t}, z, \alpha ; q\right)=\frac{(1-q)^{-1}}{(q ; q)_{\infty}} \int_{0}^{1} w^{t-1}(w q ; q)_{\infty} \psi_{0}(0, z, a w ; q) d_{q} w$ 
Ramanujan's fifth order and tenth order mock theta functions - a ...285

(iv) $\quad F_{0}\left(q^{t}, z, \alpha ; q\right)=\frac{(1-q)^{-1}}{(q ; q)_{\infty}} \int_{0}^{1} w^{t-1}(w q ; q)_{\infty} F_{0}(0, z, a w ; q) d_{q} w$

(v) $\quad \chi_{0}\left(q^{t}, z, \alpha ; q\right)=\frac{(1-q)^{-1}}{(q ; q)_{\infty}} \int_{0}^{1} w^{t-1}(w q ; q)_{\infty} \chi_{0}(0, z, a w ; q) d_{q} w$

(vi) $\quad f_{1}\left(q^{t}, z, \alpha ; q\right)=\frac{(1-q)^{-1}}{(q ; q)_{\infty}} \int_{0}^{1} w^{t-1}(w q ; q)_{\infty} f_{1}(0, z, a w ; q) d_{q} w$

(vii) $\quad \varphi_{1}\left(q^{t}, z, \alpha ; q\right)=\frac{(1-q)^{-1}}{(q ; q)_{\infty}} \int_{0}^{1} w^{t-1}(w q ; q)_{\infty} \varphi_{1}(0, z, a w ; q) d_{q} w$

(viii) $\quad \psi_{1}\left(q^{t}, z, \alpha ; q\right)=\frac{(1-q)^{-1}}{(q ; q)_{\infty}} \int_{0}^{1} w^{t-1}(w q ; q)_{\infty} \psi_{1}(0, z, a w ; q) d_{q} w$

(ix) $\quad F_{1}\left(q^{t}, z, \alpha ; q\right)=\frac{(1-q)^{-1}}{(q ; q)_{\infty}} \int_{0}^{1} w^{t-1}(w q ; q)_{\infty} F_{1}(0, z, a w ; q) d_{q} w$

(x) $\quad \chi_{1}\left(q^{t}, z, \alpha ; q\right)=\frac{(1-q)^{-1}}{(q ; q)_{\infty}} \int_{0}^{1} w^{t-1}(w q ; q)_{\infty} \chi_{1}(0, z, a w ; q) d_{q} w$.

A detailed proof for $f_{0}\left(q^{t}, z, \alpha ; q\right)$ is given. The proofs of the other functions are similar, so omitted.

Proof

By definition

$$
f_{0}(t, z, \alpha ; q)=\frac{1}{(t)_{\infty}} \sum_{n=0}^{\infty} \frac{(t)_{n} q^{n^{2}-3 n+n \alpha} z^{2 n}}{(-z ; q)_{n}}
$$

Replacing $t$ by $q^{t}$ and $q^{\alpha}$ by $a$, we have

$$
\begin{aligned}
f_{0}\left(q^{t}, z, \alpha ; q\right) & =\frac{1}{\left(q^{t}\right)_{\infty}} \sum_{n=0}^{\infty} \frac{\left(q^{t}\right)_{n} q^{n^{2}-3 n+n \alpha} z^{2 n}}{(-z ; q)_{n}} \\
& =\sum_{n=0}^{\infty} \frac{q^{n^{2}-3 n+n \alpha} z^{2 n}}{(-z ; q)_{n}\left(q^{n+t}\right)_{\infty}} \\
& =\sum_{n=0}^{\infty} \frac{q^{n^{2}-3 n+n \alpha} z^{2 n}}{(-z ; q)_{n}} \frac{(1-q)^{-1}}{(q ; q)_{\infty}} \int_{0}^{1} w^{n+t-1}(w q ; q)_{\infty} d_{q} w
\end{aligned}
$$

by $(5.1)$

$$
=\frac{(1-q)^{-1}}{(q ; q)_{\infty}} \int_{0}^{1} w^{t-1}(w q ; q)_{\infty} d_{q} w \sum_{n=0}^{\infty} \frac{q^{n^{2}-3 n} z^{2 n}}{(-z ; q)_{n}}(a w)^{n} d_{q} w
$$

But 


$$
f_{0}(0, z, \alpha ; q)=\sum_{n=0}^{\infty} \frac{q^{n^{2}-3 n+n \alpha} z^{2 n}}{(-z ; q)_{n}}
$$

and since $q^{\alpha}=a$,

$$
f_{0}(0, z, a ; q)=\sum_{n=0}^{\infty} \frac{(a)^{n} q^{n^{2}-3 n} z^{2 n}}{(-z ; q)_{n}} .
$$

Hence

$$
f_{0}(0, z, a w ; q)=\sum_{n=0}^{\infty} \frac{q^{n^{2}-3 n} z^{2 n}(a w)^{n}}{(-z ; q)_{n}} .
$$

By (5.3), (5.2) can be written as

$$
f_{0}\left(q^{t}, z, \alpha ; q\right)=\frac{(1-q)^{-1}}{(q ; q)_{\infty}} \int_{0}^{1} w^{t-1}(w q ; q)_{\infty} f_{0}(0, z, a w ; q) d_{q} w
$$

which proves Theorem 3(i). The proof of all the other fuctions is similar, so omitted.

\section{Multibasic expansion of generalized fifth order mock theta functions}

Using the summation formula [5, (3.6.7), p.71] and [6, Lemma 10, p.57], we have the multibasic expansion

$$
\begin{gathered}
\sum_{k=0}^{\infty} \frac{\left(1-a p^{k} q^{k}\right)\left(1-b p^{k} q^{-k}\right)(a, b ; p)_{k}(c, a / b c ; q)_{k} q^{k}}{(1-a)(1-b)(q, a q / b ; q)_{k}(a p / c, b c p ; p)_{k}} \sum_{m=0}^{\infty} \alpha_{m+k} \\
=\sum_{m=0}^{\infty} \frac{(a p, b p ; p)_{m}(c q, a q / b c ; q)_{m}}{(a p / c, b c p ; p)_{m}(q, a q / b ; q)_{m}} \alpha_{m} .
\end{gathered}
$$

\section{Corollary 1}

Letting $q \rightarrow q^{2}$ and $c \rightarrow \infty$ in (6.1), we have

$$
\sum_{k=0}^{\infty} \frac{\left(1-a p^{k} q^{2 k}\right)\left(1-b p^{k} q^{-2 k}\right)(a, b ; p)_{k} q^{k^{2}+k}}{(1-a)(1-b)\left(q^{2}, a q^{2} / b ; q^{2}\right)_{k} b^{k} p^{\frac{k^{2}+k}{2}}} \sum_{m=0}^{\infty} \alpha_{m+k}
$$


Ramanujan's fifth order and tenth order mock theta functions - a ...287

$$
=\sum_{m=0}^{\infty} \frac{(a p, b p ; p)_{m} q^{m^{2}+m}}{\left(q^{2}, a q^{2} / b ; q^{2}\right)_{m} b^{m} p^{\frac{m^{2}+m}{2}}} \alpha_{m} .
$$

\section{Corollary 2}

Letting $q \rightarrow q^{3}$ and $c \rightarrow \infty$ in (6.1), we have

$$
\begin{gathered}
\sum_{k=0}^{\infty} \frac{\left(1-a p^{k} q^{3 k}\right)\left(1-b p^{k} q^{-3 k}\right)(a, b ; p)_{k} q^{\frac{3 k^{2}+3 k}{2}}}{(1-a)(1-b)\left(q^{3}, a q^{3} / b ; q^{3}\right)_{k} b^{k} p^{\frac{k^{2}+k}{2}}} \sum_{m=0}^{\infty} \alpha_{m+k} \\
=\sum_{m=0}^{\infty} \frac{(a p, b p ; p)_{m} q^{\frac{3 m^{2}+3 m}{2}}}{\left(q^{3}, a q^{3} / b ; q^{3}\right)_{m} b^{m} p^{\frac{m^{2}+m}{2}}} \alpha_{m} .
\end{gathered}
$$

\section{Corollary 3}

Letting $q \rightarrow q^{5}$ and $c \rightarrow \infty$ in (6.1), we have

$$
\begin{gathered}
\sum_{k=0}^{\infty} \frac{\left(1-a p^{k} q^{5 k}\right)\left(1-b p^{k} q^{-5 k}\right)(a, b ; p)_{k} q^{\frac{5 k^{2}+5 k}{2}}}{(1-a)(1-b)\left(q^{5}, a q^{5} / b ; q^{5}\right)_{k} b^{k} p^{\frac{k^{2}+k}{2}}} \sum_{m=0}^{\infty} \alpha_{m+k} \\
=\sum_{m=0}^{\infty} \frac{(a p, b p ; p)_{m} q^{\frac{5 m^{2}+5 m}{2}}}{\left(q^{5}, a q^{5} / b ; q^{5}\right)_{m} b^{m} p^{\frac{m^{2}+m}{2}}} \alpha_{m} .
\end{gathered}
$$

We use following standard notation for multibasic hypergeometric series

$$
\begin{gathered}
\phi\left[\begin{array}{l}
a_{1}, \ldots . ., a_{r}: c_{1,1}, \ldots \ldots \ldots, c_{1, r_{1}}: \ldots \ldots . c_{m, 1}, \ldots \ldots \ldots ., c_{m, r_{m}} \\
b_{1}, \ldots \ldots, b_{s}: e_{1,1}, \ldots \ldots . ., e_{1, s_{1}} \ldots \ldots . e_{m, 1}, \ldots \ldots \ldots, e_{m, s_{m}}
\end{array} ; q, q_{\left.1, \ldots \ldots . ., q_{m} ; z\right]}\right. \\
=\sum_{n=0}^{\infty} \frac{\left(a_{1}, \ldots \ldots ., a_{r} ; q\right)_{n}}{\left(q, b_{1}, \ldots \ldots ., b_{s} ; q\right)_{n}} z^{n}\left[(-1)^{n} q^{\frac{n^{2}-n}{2}}\right]^{1+s-r} \prod_{j=1}^{m} \frac{\left(c_{j, 1}, \ldots \ldots, c_{j, r_{j}} ; q_{j}\right)_{n}}{\left(e_{j, 1}, \ldots \ldots ., e_{j, s_{j}} ; q_{j}\right)_{n}}\left[(-1)^{n} q_{j}^{\frac{n^{2}-n}{2}}\right]^{s_{j}-r_{j}} .
\end{gathered}
$$

\section{Theorem 4}

The generalized functions have the following expressions as the multibasic hypergeometric series :

(i) $\mathrm{f}_{0}(t, z, \alpha ; q)=\frac{1}{(t)_{\infty}} \sum_{k=0}^{\infty} \frac{\left(1-t q^{4 k-1}\right)\left(1-q^{-2 k+2}\right)(t ; q)_{k-1} q^{k^{2}-3 k+k \alpha} z^{2 k}}{\left(1-q^{k+2}\right)(z ; q)_{k}}$ 


$$
\times \phi\left[\begin{array}{l}
q, 0,0: t q^{3 k}, q^{3 k+3} \\
q^{k+3},-z q^{k}: 0,0
\end{array} ; q, q^{3} ; z^{2} q^{\alpha-2}\right]
$$

(ii)

$$
\begin{aligned}
\varphi_{0}(t, z, \alpha ; q)= & \frac{1}{(t)_{\infty}} \sum_{k=0}^{\infty} \frac{\left(1-t q^{4 k-1}\right)\left(1-q^{-2 k+1}\right)(t ; q)_{k-1}\left(-q^{3} / z^{2} ; q^{2}\right)_{k} q^{k^{2}+k+k \alpha}}{\left(1-q^{k+1}\right) z^{2 k}} \\
& \times \phi\left[\begin{array}{l}
q, 0:-q^{2 k+3} / z^{2}: t q^{3 k+1}, q^{3 k+3} \\
q^{k+2}: 0: 0,0
\end{array} ;, q^{2}, q^{3} ; z^{-2} q^{\alpha+1}\right]
\end{aligned}
$$

(iii)

$$
\begin{aligned}
\psi_{0}(t, z, \alpha ; q)=\frac{1}{(t)_{\infty}} & \sum_{k=0}^{\infty} \frac{\left(1-t q^{3 k-1}\right)\left(1-q^{-k+1}\right)(t ; q)_{k-1}\left(-q^{2} / z ; q\right)_{k-1} q^{\frac{k(k+1)}{2}+k \alpha}}{\left(1-q^{k+1}\right) z^{k}} \\
& \times \phi\left[\begin{array}{l}
q,-q^{k+1} / z: t q^{2 k}, q^{2 k+2} \\
q^{k+2}: 0,0
\end{array}\right.
\end{aligned}
$$

(iv)

$$
\begin{gathered}
F_{0}(t, z, \alpha ; q)=\frac{1}{(t)_{\infty}} \sum_{k=0}^{\infty} \frac{\left(1-t q^{6 k-1}\right)\left(1-q^{-4 k+4}\right)(t ; q)_{k-1} q^{2 k^{2}-5 k+k \alpha} z^{4 k}}{\left(1-q^{k+4}\right)\left(z^{2} / q ; q^{2}\right)_{k}} \\
\quad \times \phi\left[\begin{array}{l}
q, 0: 0: t 5^{5 k}, q^{5 k+5} \\
q^{k+5}: z^{2} q^{2 k-1}: 0,0
\end{array} ;, q^{2}, q^{5} ; z^{4} q^{\alpha-3}\right]
\end{gathered}
$$

(v)

$$
\begin{aligned}
f_{1}(t, z, \alpha ; q)=\frac{1}{(t)_{\infty}} & \sum_{k=0}^{\infty} \frac{\left(1-t q^{4 k-1}\right)\left(1-q^{-2 k+1}\right)(t ; q)_{k-1} q^{k^{2}-2 k+k \alpha} z^{2 k}}{\left(1-q^{k+1}\right)(-z ; q)_{k}} \\
& \times \phi\left[\begin{array}{l}
q, 0,0: t q^{3 k+1}, q^{3 k+3} \\
q^{k+2},-z q^{k}: 0,0
\end{array} ;, q^{3} ; z^{2} q^{\alpha-2}\right]
\end{aligned}
$$

(vi) $\varphi_{1}(t, z, \alpha ; q)$

$$
\begin{aligned}
=\frac{z^{2}}{q^{2}} \frac{1}{(t)_{\infty}} \sum_{k=0}^{\infty} & \frac{\left(1-t q^{4 k-1}\right)\left(1-q^{-2 k+1}\right)(t ; q)_{k-1}\left(-q^{3} / z^{2} ; q^{2}\right)_{k-1} q^{k^{2}+k+k \alpha}}{\left(1-q^{k+1}\right) z^{2 k}} \\
& \times \phi\left[\begin{array}{l}
q, 0:-q^{2 k+1} / z^{2}: t q^{3 k+1}, q^{3 k+3} \\
q^{k+2}: 0: 0,0
\end{array} ; q, q^{2}, q^{3} ; z^{-2} q^{\alpha+1}\right]
\end{aligned}
$$


Ramanujan's fifth order and tenth order mock theta functions - a ...289

(vii)

$$
\begin{aligned}
\psi_{1}(t, z, \alpha ; q)=\frac{q}{z} & \frac{1}{(t)_{\infty}} \sum_{k=0}^{\infty} \frac{\left(1-t q^{3 k-1}\right)\left(1-q^{-k+1}\right)(t ; q)_{k-1}\left(-q^{2} / z ; q\right)_{k} q^{\frac{k(k+1)}{2}+k \alpha}}{\left(1-q^{k+1}\right)(z ; q)_{k}} \\
& \times \phi\left[\begin{array}{l}
q,-q^{k+2} / z: t q^{2 k}, q^{2 k+2} \\
q^{k+2}: 0,0
\end{array} ;, q^{2} ; z^{-1} q^{\alpha+1}\right]
\end{aligned}
$$

(viii)

$$
\begin{aligned}
F_{1}(t, z, \alpha ; q)= & \frac{1}{(t)_{\infty}} \sum_{k=0}^{\infty} \frac{\left(1-t q^{6 k-1}\right)\left(1-q^{-4 k+4}\right)(t ; q)_{k-1} q^{2 k^{2}-3 k+k \alpha} z^{4 k}}{\left(1-q^{k+4}\right)\left(z^{2} / q ; q^{2}\right)_{k+1}} \\
& \times \phi\left[\begin{array}{l}
q, 0: 0: t q^{5 k}, q^{k+5} \\
q^{k+5}: z^{2} q^{2 k+1}: 0,0
\end{array} ; q, q^{2}, q^{5} ; z^{4} q^{\alpha-1}\right]
\end{aligned}
$$

We shall give the proof of (i) only, for others we will state the value of the parameters.

\section{Proof of (i)} $(6.3)$,

Taking $a=t / q, b=q^{2}, p=q$ and $\alpha_{m}=\frac{q^{m \alpha-2 m}\left(q^{3} ; q^{3}\right)_{m}\left(t ; q^{3}\right)_{m} z^{2 m}}{\left(q^{3} ; q\right)_{m}(-z ; q)_{m}}$ in we have

$$
\begin{aligned}
& \sum_{k=0}^{\infty} \frac{\left(1-t q^{4 k-1}\right)\left(1-q^{-2 k+2}\right)\left(t / q, q^{2} ; q\right)_{k} q^{\frac{3 k^{2}+3 k}{2}}}{(1-t / q)\left(1-q^{2}\right)\left(t, q^{3} ; q^{3}\right)_{k} q^{\frac{k^{2}+5 k}{2}}} \\
& \times \sum_{m=0}^{\infty} \frac{\left(q^{3} ; q^{3}\right)_{m+k}\left(t ; q^{3}\right)_{m+k} z^{2(m+k)} q^{(m+k) \alpha-2(m+k)}}{\left(q^{3} ; q\right)_{m+k}(-z ; q)_{m+k}} \\
& =\sum_{m=0}^{\infty} \frac{q^{m^{2}-3 m+m \alpha}(t ; q)_{m} z^{2 m}}{(-z ; q)_{m}}
\end{aligned}
$$

The right hand side of (6.5) is equal to

$$
(t, q)_{\infty} f_{0}(t, z, \alpha ; q)
$$

The left hand side of (6.5) is equal to

$$
\sum_{k=0}^{\infty} \frac{\left(1-t q^{4 k-1}\right)\left(1-q^{-2 k+2}\right)(t ; q)_{k-1}\left(q^{2} ; q\right)_{k}\left(q^{3} ; q^{3}\right)_{k}\left(t ; q^{3}\right)_{k} q^{k^{2}-3 k+k \alpha} z^{2 k}}{\left(1-q^{2}\right)\left(q^{3}, t ; q^{3}\right)_{k}\left(q^{3} ; q\right)_{k}(-z ; q)_{k}}
$$




$$
\begin{aligned}
& \times \sum_{m=0}^{\infty} \frac{q^{m \alpha-2 m}\left(q^{3 k+3} ; q^{3}\right)_{m}\left(t q^{3 k} ; q^{3}\right)_{m} z^{2 m}}{\left(q^{k+3} ; q\right)_{m}\left(-z q^{k} ; q\right)_{m}} \\
& =\sum_{k=0}^{\infty} \frac{\left(1-t q^{4 k-1}\right)\left(1-q^{-2 k+2}\right)(t ; q)_{k-1} q^{k^{2}-3 k+k \alpha} z^{2 k}}{\left(1-q^{k+2}\right)(-z ; q)_{k}} \\
& \times\left[\begin{array}{l}
q, 0,0: t t^{3 k}, q^{3 k+3} \\
q^{k+3},-z q^{k}: 0,0
\end{array} ; q, q^{3} ; z^{2} q^{\alpha-2}\right],
\end{aligned}
$$

which proves Theorem 4 (i).

\section{Proof of (ii)}

Take $a=t / q, b=q, p=q$ and

$\alpha_{m}=\frac{q^{m \alpha+m}\left(q^{3} ; q^{3}\right)_{m}\left(t q ; q^{3}\right)_{m}\left(-q^{2} / z^{2} ; q^{2}\right)_{m}}{\left(q^{2} ; q\right)_{m} z^{2 m}}$ in (6.3).

\section{Proof of (iii)}

Take $a=t / q, b=q, p=q$ and $\alpha_{m}=\frac{q^{m \alpha+m}\left(q^{2} ; q^{2}\right)_{m}\left(t ; q^{2}\right)_{m}\left(-q^{2} / z ; q\right)_{m-1}}{\left(q^{2} ; q\right)_{m} z^{m}}$ in (6.2).

\section{Proof of (iv)}

Take $a=t / q, b=q^{4}, p=q$ and $\alpha_{m}=\frac{q^{m \alpha-3 m}\left(q^{5} ; q^{5}\right)_{m}\left(t ; q^{5}\right)_{m} z^{4 m}}{\left(q^{5} ; q\right)_{m}\left(z^{2} / q ; q^{2}\right)_{m}}$ in (6.4).

\section{Proof of (v)}

Take $a=t / q, b=q, p=q$ and $\alpha_{m}=\frac{q^{m \alpha-2 m}\left(q^{3} ; q^{3}\right)_{m}\left(t q ; q^{3}\right)_{m} z^{2 m}}{\left(q^{2} ; q\right)_{m}(-z ; q)_{m}}$ in $(6.3)$.

\section{Proof of (vi)}

Take $a=t / q, b=q, p=q$ and $\alpha_{m}=\frac{q^{m \alpha+m}\left(q^{3} ; q^{3}\right)_{m}\left(t q ; q^{3}\right)_{m}\left(-q^{3} / z^{2} ; q^{2}\right)_{m-1}}{\left(q^{2} ; q\right)_{m} z^{2 m}}$ in (6.3).

\section{Proof of (vii)}

Take $a=t / q, b=q, p=q$ and $\alpha_{m}=\frac{q^{m \alpha+m}\left(q^{2} ; q^{2}\right)_{m}\left(t ; q^{2}\right)_{m}\left(-q^{2} / z ; q\right)_{m}}{\left(q^{2} ; q\right)_{m} z^{m}}$ in (6.2).

\section{Proof of (viii)}


Take $a=t / q, b=q^{4}, p=q$ and $\alpha_{m}=\frac{q^{m \alpha-m}\left(q^{5} ; q^{5}\right)_{m}\left(t ; q^{5}\right)_{m} z^{4 m}}{\left(q^{5} ; q\right)_{m}\left(z^{2} / q ; q^{2}\right)_{m+1}}$ in (6.4).

\section{Generalized tenth order mock theta functions are $F_{q}$-functions}

We show that the four generalized tenth order mock theta functions are $F_{q^{-}}$functions.

\section{Theorem 5}

The generalized four tenth order mock theta functions $\Phi(t, z, \alpha ; q), \Psi(t, z, \alpha ; q), X(t, z, \alpha ; q)$, and $\chi(t, z, \alpha ; q)$ are $F_{q}$-functions.

We shall give a detailed proof for the function $\Phi(t, z, \alpha ; q)$, the proof for the other functions are similar, so omitted.

\section{Proof}

Apply the difference oprator $D_{q, t}$ to $\Phi(t, z, \alpha ; q)$, we have $t D_{q, t} \Phi(t, z, \alpha ; q)=\Phi(t, z, \alpha ; q)-\Phi(t q, z, \alpha ; q)$

$=\frac{1}{(t)_{\infty}} \sum_{n=0}^{\infty} \frac{(t)_{n} q^{n(n-3) / 2+n \alpha} z^{n}}{\left(z^{2} / q ; q^{2}\right)_{n+1}}-\frac{1}{(t q)_{\infty}} \sum_{n=0}^{\infty} \frac{(t q)_{n} q^{n(n-3) / 2+n \alpha} z^{n}}{\left(z^{2} / q ; q^{2}\right)_{n+1}}$

$=\frac{1}{(t)_{\infty}} \sum_{n=0}^{\infty} \frac{(t)_{n} q^{n(n-3) / 2+n \alpha} z^{n}}{\left(z^{2} / q ; q^{2}\right)_{n+1}}-\frac{1}{(t)_{\infty}} \sum_{n=0}^{\infty} \frac{(t)_{n} q^{n(n-3) / 2+n \alpha+n} z^{n}\left(1-t q^{n}\right)}{\left(z^{2} / q ; q^{2}\right)_{n+1}}$ $=\frac{t}{(t)_{\infty}} \sum_{n=0}^{\infty} \frac{(t)_{n} q^{n(n-3) / 2+n(\alpha+1)} z^{n}}{\left(z^{2} / q ; q\right)_{n+1}}$.

So

$$
D_{q, t} \Phi(t, z, \alpha ; q),=\Phi(t, z, \alpha+1 ; q) \text {. }
$$

Hence $\Phi(t, z, \alpha ; q)$ is a $F_{q}$-function. Similarly the other three functions are also $F_{q}$-functions.

\section{Relations between generalized mock theta function of tenth order}

For proving the relationship between these generalized functions we shall use the property that they are $F_{q}$-functions.

\section{Theorem 6}


(i) $D_{q, t} \Phi(t, z, \alpha ; q)=\frac{1}{z} \Psi(t, z, \alpha ; q)$.

(ii) $X(t, z, \alpha ; q)=\frac{1}{(t)_{\infty}} \sum_{n=0}^{\infty} \frac{(-1)^{n}(t)_{n} q^{n^{2}-3 n+n \alpha} z^{2 n}}{\left(-z^{2} / q ; q\right)_{2 n+1}}+\frac{z^{2}}{q^{2}} \chi(t, z, \alpha ; q)$.

Proof of (i)

$$
\begin{aligned}
D_{q, t} \Phi(t, z, \alpha ; q)= & \Phi(t, z, \alpha+1 ; q) \\
& =\frac{1}{(t)_{\infty}} \sum_{n=0}^{\infty} \frac{(t)_{n} q^{n(n-3) / 2+n \alpha+n} z^{n}}{\left(z^{2} / q ; q^{2}\right)_{n+1}} \\
& =\frac{1}{(t)_{\infty}} \sum_{n=0}^{\infty} \frac{(t)_{n} q^{n(n-1) / 2+n \alpha} z^{n}}{\left(z^{2} / q ; q^{2}\right)_{n+1}} \\
& =\frac{1}{z} \Psi(t, z, \alpha ; q),
\end{aligned}
$$

which proves Theorem 6(i).

\section{Proof of (ii)}

$$
\begin{aligned}
X(t, z, \alpha ; q)= & \frac{1}{(t)_{\infty}} \sum_{n=0}^{\infty} \frac{(-1)^{n}(t)_{n} q^{n^{2}-3 n+n \alpha} z^{2 n}\left(1+z^{2} q^{2 n-1}\right)}{\left(-z^{2} / q ; q\right)_{2 n+1}} \\
& =\frac{1}{(t)_{\infty}} \sum_{n=0}^{\infty} \frac{(-1)^{n}(t)_{n} q^{n^{2}-3 n+n \alpha} z^{2 n}}{\left(-z^{2} / q ; q\right)_{2 n+1}}+\frac{z^{2}}{q} \frac{1}{(t)_{\infty}} \\
& \times \sum_{n=0}^{\infty} \frac{(-1)^{n}(t)_{n} q^{n^{2}-n+n \alpha} z^{2 n}}{\left(-z^{2} / q ; q\right)_{2 n+1}} \\
= & \frac{1}{(t)_{\infty}} \sum_{n=0}^{\infty} \frac{(-1)^{n}(t)_{n} q^{n^{2}-3 n+n \alpha} z^{2 n}}{\left(-z^{2} / q ; q\right)_{2 n+1}}+\frac{z^{2}}{q^{2}} \chi(t, z, \alpha ; q),
\end{aligned}
$$

which proves Theorem 6(ii).

\section{9. $q$-Integral representation for the generalized tenth order mock theta functions}

We give $q$-integral representation for the four generalized tenth order mock theta functions.

\section{Theorem 7}

The following equalities hold :

(i) $\Phi\left(q^{t}, z, \alpha ; q\right)=\frac{(1-q)^{-1}}{(q ; q)_{\infty}} \int_{0}^{1} w^{t-1}(w q ; q)_{\infty} \Phi(0, z, a w ; q) d_{q} w$

(ii) $\Psi\left(q^{t}, z, \alpha ; q\right)=\frac{(1-q)^{-1}}{(q ; q)_{\infty}} \int_{0}^{1} w^{t-1}(w q ; q)_{\infty} \Psi(0, z, a w ; q) d_{q} w$

(iii) $X\left(q^{t}, z, \alpha ; q\right)=\frac{(1-q)^{-1}}{(q ; q)_{\infty}} \int_{0}^{1} w^{t-1}(w q ; q)_{\infty} X(0, z, a w ; q) d_{q} w$ 
Ramanujan's fifth order and tenth order mock theta functions - a ...293

(iv) $\chi\left(q^{t}, z, \alpha ; q\right)=\frac{(1-q)^{-1}}{(q ; q)_{\infty}} \int_{0}^{1} w^{t-1}(w q ; q)_{\infty} \chi(0, z, a w ; q) d_{q} w$.

We give a detailed proof for $\Phi\left(q^{t}, z, \alpha ; q\right)$ only and omit the proof for the other functions, as they are similar.

Proof

By definition

$$
\Phi(t, z, \alpha ; q)=\frac{1}{(t)_{\infty}} \sum_{n=0}^{\infty} \frac{(t)_{n} q^{n(n-3) / 2+n \alpha} z^{n}}{\left(z^{2} / q ; q^{2}\right)_{n+1}}
$$

Replacing $t$ by $q^{t}$ and $q^{\alpha}$ by $a$, we have

$$
\begin{aligned}
\Phi\left(q^{t}, z, \alpha ; q\right)= & \frac{1}{\left(q^{t}\right)_{\infty}} \sum_{n=0}^{\infty} \frac{\left(q^{t}\right)_{n} q^{n(n-3) / 2+n \alpha} z^{n}}{\left(z^{2} / q ; q^{2}\right)_{n+1}} \\
& =\sum_{n=0}^{\infty} \frac{q^{n(n-3) / 2+n \alpha} z^{n}}{\left(z^{2} / q ; q^{2}\right)_{n+1}\left(q^{n+t}\right)_{\infty}} \\
& =\sum_{n=0}^{\infty} \frac{q^{n(n-3) / 2+n \alpha} z^{n}}{(1-q)^{-1}} \frac{\left(z^{2} / q ; q^{2}\right)_{n+1}}{(q ; q)_{\infty}} \int_{0}^{1} w^{n+t-1}(w q ; q)_{\infty} d_{q} w
\end{aligned}
$$

by $(5.1)$

$$
=\frac{(1-q)^{-1}}{(q ; q)_{\infty}} \int_{0}^{1} w^{t-1}(w q ; q)_{\infty} \sum_{n=0}^{\infty} \frac{q^{n(n-3) / 2+n \alpha} z^{n}}{\left(z^{2} / q ; q^{2}\right)_{n+1}}(a w)^{n} d_{q} w
$$

But

$$
\Phi(0, z, \alpha ; q)=\sum_{n=0}^{\infty} \frac{q^{n(n-3) / 2+n \alpha} z^{n}}{\left(z^{2} / q ; q^{2}\right)_{n+1}}
$$

and since $q^{\alpha}=a$,

$$
\Phi(0, z, \alpha ; q)=\sum_{n=0}^{\infty} \frac{(a)^{n} q^{n(n-3) / 2} z^{n}}{\left(z^{2} / q ; q^{2}\right)_{n+1}}
$$

Hence

$$
\Phi(0, z, a w ; q)=\sum_{n=0}^{\infty} \frac{q^{n(n-3) / 2}(a w)^{n}}{\left(z^{2} / q ; q^{2}\right)_{n+1}}
$$

By (9.2), (9.1) can be written as

$$
\Phi\left(q^{t}, z, \alpha ; q\right)=\frac{(1-q)^{-1}}{(q ; q)_{\infty}} \int_{0}^{1} w^{t-1}(w q ; q)_{\infty} \Phi(0, z, a w ; q) d_{q} w
$$


which proves Theorem 7 (i). The proof for the other three functions being similar, so omitted.

\section{Multibasic expansions for generalized tenth order mock theta functions}

We now give multibasic expansions for our generalized functions.

\section{Theorem 8}

The generalized functions have the following expressions as a multibasic hypergeometric series:

(i) $\Phi(t, z, \alpha ; q)=$

$$
\begin{gathered}
\frac{\left(1-z^{2} / q\right)^{-1}}{(t ; q)_{\infty}} \sum_{k=0}^{\infty} \frac{\left(1-t q^{3 k-1}\right)\left(1-q^{-k+2}\right)(t ; q)_{k-1} q^{\frac{k(k-3)}{2}+k \alpha} z^{k}}{\left(1-q^{k+2}\right)\left(z^{2} q ; q^{2}\right)_{k}} \\
\times \phi\left[\begin{array}{l}
q, 0: t q^{2 k-1}, q^{2 k+2} \\
q^{k+3}: z^{2} q^{2 k+1}, 0
\end{array} ; q, q^{2} ; z q^{\alpha}\right]
\end{gathered}
$$

(ii) $\Psi(t, z, \alpha ; q)=$

$$
\begin{aligned}
& \frac{z\left(1-z^{2} / q\right)^{-1}}{(t ; q)_{\infty}} \sum_{k=0}^{\infty} \frac{\left(1-t q^{3 k-1}\right)\left(1-q^{-k+1}\right)(t ; q)_{k-1} q^{\frac{k(k-1)}{2}+k \alpha} z^{k}}{\left(1-q^{k+1}\right)\left(z^{2} q ; q^{2}\right)_{k}} \\
& \times \phi\left[\begin{array}{l}
q, 0: t q^{2 k}, q^{2 k+2} \\
q^{k+2}: z^{2} q^{2 k+1}, 0
\end{array} ; q, q^{2} ; z q^{\alpha}\right] \\
& \text { (iii) } X(t, z, \alpha ; q)=\frac{1}{(t ; q)_{\infty}} \sum_{k=0}^{\infty} \frac{(-1)^{k}\left(1-q^{-2 k+2}\right)(t ; q)_{k} q^{k^{2}-3 k+k \alpha} z^{2 k}}{\left(1-q^{k+2}\right)\left(-z^{2} / q ; q\right)_{2 k}} \\
& \times \phi\left[\begin{array}{l}
q, t q^{k}: 0,0: q^{3 k+3} \\
q^{k+3}:-z^{2} q^{2 k-1},-z^{2} q^{2 k}: 0
\end{array} ; q, q^{2}, q^{3} ;-z^{2} q^{\alpha-2}\right] \\
& \text { (iv) } \quad \chi(t, z, \alpha ; q)=\frac{q}{(t ; q)_{\infty}} \sum_{k=0}^{\infty} \frac{(-1)^{k}\left(1-q^{1-2 k}\right)(t ; q)_{k} q^{k^{2}-k+k \alpha} z^{2 k}}{\left(1-q^{2 k+1}\right)\left(-z^{2} / q ; q\right)_{2 k+1}} \\
& \times \phi\left[\begin{array}{l}
q, t q^{k}: 0,0: q^{3 k+3} \\
q^{k+2}:-z^{2} q^{2 k},-z^{2} q^{2 k+1}: 0
\end{array} ; q, q^{2}, q^{3} ;-z^{2} q^{\alpha-1}\right] .
\end{aligned}
$$

\section{Proof of (i)}

Take $a=t / q, b=q^{2}, p=q$ and $\alpha_{m}=\frac{q^{m \alpha}\left(q^{2} ; q^{2}\right)_{m}\left(t / q ; q^{2}\right)_{m} z^{m}}{\left(q^{3} ; q\right)_{m}\left(z^{2} q ; q^{2}\right)_{m}}$ in (6.2).

\section{Proof of (ii)}


Ramanujan's fifth order and tenth order mock theta functions - a ...295

Take $a=t / q, b=q, p=q$ and $\alpha_{m}=\frac{q^{m \alpha}\left(q^{2} ; q^{2}\right)_{m}\left(t ; q^{2}\right)_{m} z^{m}}{\left(q^{2} ; q\right)_{m}\left(z^{2} q ; q^{2}\right)_{m}}$ in (6.2).

\section{Proof of (iii)} $(6.3)$.

Let $a \rightarrow 0, b=q^{2}, p=q$ and $\alpha_{m}=\frac{\left(-q^{\alpha-2}\right)^{m}\left(q^{3} ; q^{3}\right)_{m}(t ; q)_{m} z^{2 m}}{\left(q^{3} ; q\right)_{m}\left(-z^{2} / q ; q^{2}\right)_{2 m}}$ in

\section{Proof of (iv)} (6.3).

Let $a \rightarrow 0, b=q, p=q$ and $\alpha_{m}=\frac{\left(-q^{\alpha-1}\right)^{m}\left(q^{3} ; q^{3}\right)_{m}(t ; q)_{m} z^{2 m}}{\left(q^{2} ; q\right)_{m}\left(-z^{2} / q ; q\right)_{2 m+1}}$ in

\section{Conclusion}

Seventh order mock theta functions were more diffcult, however, we generalized them and found interesting properities. The paper has already been communicated for publication.

\section{References}

[1] G. E. Andrews, The fifth and seventh order mock theta, Trans. Amer. Math. Soc. 293, pp. 113-134, (1986).

[2] G. E. Andrews, Mock Theta Functions, Proc. Sympos. Pure Math., 49, Part 2, pp. 283-298, (1989).

[3] Youn-Seo Choi, Tenth order mock theta functions in Ramanujan's 'Lost' Notebook, Invent. Math. 136, pp. 497-569, (1999).

[4] Youn-Seo Choi, The basic bilateral hypergeometric series and the mock theta functions, Ramanujan J. 24, pp. 345-386, (2011).

[5] G. Gasper and M. Rahman, Basic Hypergeometric Series, Cambridge University Press, Cambridge, (1990).

[6] E. D. Rainville, Special Function, Chelsea Publishing Company, Bronx, New York, (1960). 
[7] S. Ramanujan, Collected Paper, Cambridge University Press 1927, reprinted by Chelsea New York, (1962).

[8] L. J. Rogers, Second memoir on the expansion of certain infinite products, Proc. London Math. Soc. 25, pp. 318-343, (1984).

[9] G. N. Watson, The final problem: An account of the mock theta functions, J. London Math. Soc. 11, pp. 55-80, (1936).

[10] G. N. Watson, The mock theta functions (2), Proc. London Math. Soc. (2) 42, pp. 274-304, (1937).

\section{Bhaskar Srivastava}

Department of Mathematics and Astronomy

University of Lucknow

Lucknow,

India

e-mail : bhaskarsrivastav@yahoo.com 